\title{
Average and Variation of Oocyte Counts in Women Undergoing in Vitro Fertilization
}

\author{
Nidenori Negoro $^{1}$, Keiichi Kumasawa ${ }^{2,}{ }^{*}$, Eri Akita ${ }^{1}$, Toshihiko Tomiyama ${ }^{1}$, Takeshi Taniguchi ${ }^{1}$ \\ ${ }^{1}$ Taniguchi Hospital, Osaka, Japan \\ ${ }^{2}$ The Department of Obstetrics and Gynecology, University of Tokyo, Tokyo, Japan
}

Email address:

neko_neko_nego-pi@yahoo.co.jp (N. Negoro), kokoko52@hotmail.com (K. Kumasawa), erimanboo0611@gmail.com (E. Akita), tomymd7@gmail.com (T. Tomiyama), takeshi@taniguchi-hp.org (T. Taniguchi)

${ }^{*}$ Corresponding author

\section{To cite this article:}

Nidenori Negoro, Keiichi Kumasawa, Eri Akita, Toshihiko Tomiyama, Takeshi Taniguchi. Average and Variation of Oocyte Counts in Women Undergoing in Vitro Fertilization. Journal of Gynecology and Obstetrics. Vol. 9, No. 2, 2021, pp. 31-35. doi: 10.11648/j.jgo.20210902.11

Received: January 25, 2021; Accepted: February 18, 2021; Published: March 4, 2021

\begin{abstract}
Artificial reproductive technology (ART) has become a standard treatment for patients with infertility. However the cost is burdensome to infertile patients in some countries. Moreover aging is a critical factor for infertile patients and is accompanied by higher risk of condition and counts of oocytes. In planning ART for a patient, there are two patterns of oocyte pick-up (OPU) method: One is to transfer all the embros which are obtained from one OPU. Then next OPU will be performed when she fails to get pregnant despite using up all embryos. The other method is to repeat several consecutive OPUs until sufficient stock of embryos. The sufficiency depends on age, serum AMH level, etc. This study investigates the deviation in the number of oocytes collected by OPU in infertile patients who received multiple OPU within 1 year. The number of oocytes increased as the average number of oocytes increased. However, the ratio of standard deviation/average decreased. The frequency of required OPU depends on the pregnancy rate per embryo, the background of the patient, and the number of eggs collected at the first or second OPU. Thus, the analysis will reduce their mental and economic burden. Further studies with large-scale data are needed to determine the ideal frequency of OPU.
\end{abstract}

Keywords: Infertility, Artificial Reproductive Technology (ART), Oocyte Pick up (OPU), Cost, Variation of Oocyte Count

\section{Introduction}

Artificial reproductive technology (ART) has become a standard treatment for patients with infertility. However, ovulation induction, oocyte pick-up (OPU), and embryo transfer (ET) in ART are accompanied by psychological and physical stress. Furthermore, expense of ART is higher compared to that of artificial insemination of husband (AIH), thus ART is economically burdensome for patients in some countries, including Japan. Moreover even ART can't be helpful to all the infertile patients.

When a infertile woman doesn't get pregnant, with the first ART therapy, she will go to the next ART therapy if she wants to her baby It comes to repeated therapies until successful pregnancy, leading to a large amount of cost.

To reduce the burden, there are two ways: One is to collect oocytes and sequentially transfer frozen embryos and after running out of frozen embryos, another OPU will be performed. The other is to repeat OPU until a sufficient amount of oocytes or embryos is obtained from the point of decreasing fertility due to aging and other factors, even during the cycles of frozen embryo transfer. A strategy is selected on a case-by-case basis, and the patient may choose after being informed of both methods [1].

Currently, the serum level of anti-Mullerian hormone (AMH) is adopted as a test of ovarian reserve, and it has been reported to be closely associated with follicle counts during ovulation induction $[2,3]$. A higher level of anti-Müllerian hormone when tested in women in the general population has been found to have a positive correlation with natural fertility in women aged 30-44 aiming to conceive spontaneously, even after adjusting for age $[3,4]$. Therefore, AMH is also used as a guide for determining the number of oocytes by ovulation induction $[5,6]$. However, serum AMH levels are also affected by PCOS $[7,8]$. In addition, AMH is not a suitable 
predictor of IVF success [9, 10]. Additionally, AMH levels are used to estimate a woman's remaining egg supply.

Moreover, in the past 10 years, some reports have stated that AMH itself will fluctuate such as a patient with PCOS, and we reported a case with a elevation of AMH after delivery [11]. Therefore, to predict the number of oocytes in the following OPU more precisely, we focused on the oocyte count itself in the previous OPU. To examine it, this study analyzed the variation in the number of oocytes for each infertile patient throughout multiple times within a year from the first OPU.

\section{Materials and Methods}

Among 103 infertile women who received OPU at Taniguchi Hospital from January 2017 to December 2017, only 29 women had multiple OPU within 1 year after the first OPU. The ovulation induction method and the number of oocytes in each OPU are listed in Table 1. In addition, the average and standard deviation (SD) were recorded, and the correlation between the average and SD was evaluated. Differences were assessed using t-tests in Microsoft Excel 10. P-values of $<0.05$ were considered statistically significant.

Table 1. Characteristics of ART, controlled ovarian hyperstimulation, and oocyte count for each OPU.

\begin{tabular}{|c|c|c|c|c|}
\hline patient & age & ART & controlled ovarian hyperstimulation & oocyte count \\
\hline 1 & 38 & IVF & GnRH antagonist+hMG & 3 \\
\hline 1 & 38 & IVF & GnRH antagonist+hMG & 5 \\
\hline 2 & 35 & IVF & GnRH antagonist $+\mathrm{hMG}$ & 3 \\
\hline 2 & 35 & IVF & long GnRHa+hMG & 3 \\
\hline 3 & 42 & IVF & GnRH antagonist $+\mathrm{hMG}$ & 9 \\
\hline 3 & 42 & IVF & long $\mathrm{GnRHa}+\mathrm{hMG}$ & 15 \\
\hline 3 & 42 & IVF & $\mathrm{hMG}$ & 8 \\
\hline 3 & 42 & IVF & hMG & 6 \\
\hline 4 & 36 & IVF & GnRH antagonist $+\mathrm{hMG}$ & 6 \\
\hline 4 & 37 & IVF & long GnRHa+hMG & 7 \\
\hline 4 & 37 & IVF & GnRH antagonist $+\mathrm{hMG}$ & 4 \\
\hline 5 & 38 & IVF & hMG & 2 \\
\hline 5 & 38 & IVF & GnRH antagonist $+\mathrm{hMG}$ & 4 \\
\hline 6 & 33 & IVF & GnRH antagonist+hMG & 8 \\
\hline 6 & 33 & IVF & GnRH antagonist+hMG & 7 \\
\hline 7 & 40 & IVF & GnRH antagonist $+\mathrm{hMG}$ & 5 \\
\hline 7 & 40 & IVF & GnRH antagonist+hMG & 1 \\
\hline 7 & 41 & IVF & GnRH antagonist $+\mathrm{hMG}$ & 3 \\
\hline 8 & 40 & ICSI & GnRH antagonist+hMG & 2 \\
\hline 8 & 41 & ICSI & $\mathrm{CC}$ & 1 \\
\hline 9 & 39 & ICSI & $\mathrm{hMG}$ & 2 \\
\hline 9 & 40 & ICSI & long $\mathrm{GnRHa}+\mathrm{hMG}$ & 6 \\
\hline 10 & 34 & ICSI & GnRH antagonist+hMG & 4 \\
\hline 10 & 34 & ICSI & short GnRHa+hMG & 4 \\
\hline 11 & 42 & IVF & GnRH antagonist $+\mathrm{hMG}$ & 2 \\
\hline 11 & 42 & IVF & GnRH antagonist $+\mathrm{hMG}$ & 2 \\
\hline 12 & 44 & IVF & $\mathrm{CC}+\mathrm{hMG}$ & 5 \\
\hline 12 & 45 & IVF & GnRH antagonist $+\mathrm{hMG}$ & 3 \\
\hline 13 & 33 & IVF & GnRH antagonist $+\mathrm{hMG}$ & 3 \\
\hline 13 & 33 & IVF+ICSI & long GnRHa+hMG & 4 \\
\hline 14 & 39 & ICSI & long $\mathrm{GnRHa}+\mathrm{hMG}$ & 8 \\
\hline 14 & 40 & ICSI & long $\mathrm{GnRHa}+\mathrm{hMG}$ & 8 \\
\hline 15 & 41 & ICSI & long $\mathrm{GnRHa}+\mathrm{hMG}$ & 2 \\
\hline 15 & 41 & ICSI & long $\mathrm{GnRHa}+\mathrm{hMG}$ & 3 \\
\hline 15 & 41 & ICSI & $\mathrm{CC}+\mathrm{hMG}$ & 2 \\
\hline 15 & 41 & ICSI & $\mathrm{CC}+\mathrm{GnRH}$ antagonist $+\mathrm{hMG}$ & 2 \\
\hline 15 & 41 & ICSI & $\mathrm{CC}+\mathrm{hMG}$ & 3 \\
\hline 16 & 35 & ICSI+IVF & GnRH antagonist $+\mathrm{hMG}$ & 12 \\
\hline 16 & 36 & ICSI & GnRH antagonist+hMG & 3 \\
\hline 16 & 36 & ICSI & GnRH antagonist $+\mathrm{hMG}$ & 9 \\
\hline 17 & 39 & ICSI & GnRH antagonist+hMG & 4 \\
\hline 17 & 39 & ICSI & hMG & 7 \\
\hline 18 & 42 & ICSI & long $\mathrm{GnRHa}+\mathrm{hMG}$ & 5 \\
\hline 18 & 42 & IVF & long $\mathrm{GnRHa}+\mathrm{hMG}$ & 7 \\
\hline 19 & 23 & IVF & GnRHantagonist $+\mathrm{hMG}$ & 8 \\
\hline 19 & 24 & IVF+ICSI & $\mathrm{hMG}$ & 10 \\
\hline 20 & 33 & IVF & long GnRHa+hMG & 2 \\
\hline 20 & 33 & IVF & GnRHantagonist $+\mathrm{hMG}$ & 8 \\
\hline 20 & 33 & ICSI & GnRHantagonist $+\mathrm{hMG}$ & 7 \\
\hline
\end{tabular}




\begin{tabular}{|c|c|c|c|c|}
\hline patient & age & ART & controlled ovarian hyperstimulation & oocyte count \\
\hline 21 & 35 & IVF & GnRHantagonist+hMG & 8 \\
\hline 21 & 36 & IVF & short GnRHa+hMG & 7 \\
\hline 22 & 35 & IVF & GnRHantagonist+hMG & 6 \\
\hline 22 & 36 & IVF & GnRHantagonist $+\mathrm{hMG}$ & 5 \\
\hline 23 & 39 & IVF & long GnRHa+hMG & 6 \\
\hline 23 & 40 & IVF & long $\mathrm{GnRHa}+\mathrm{hMG}$ & 7 \\
\hline 24 & 40 & IVF & long GnRHa+hMG & 4 \\
\hline 24 & 40 & IVF & GnRHantagonist $+\mathrm{hMG}$ & 4 \\
\hline 24 & 40 & IVF & GnRHantagonist $+\mathrm{hMG}$ & 3 \\
\hline 25 & 39 & IVF & long GnRHa+hMG & 8 \\
\hline 25 & 39 & IVF+ICSI & GnRHantagonist $+\mathrm{hMG}$ & 10 \\
\hline 26 & 38 & IVF & hMG & 4 \\
\hline 26 & 38 & IVF & long GnRHa+hMG & 3 \\
\hline 26 & 39 & IVF & short GnRHa+hMG & 3 \\
\hline 26 & 39 & ICSI & GnRHantagonist+hMG & 6 \\
\hline 27 & 42 & ICSI & GnRHantagonist+hMG & 0 \\
\hline 27 & 42 & IVF & GnRHantagonist+hMG & 2 \\
\hline 28 & 31 & IVF & GnRHantagonist+hMG & 4 \\
\hline 28 & 31 & ICSI & short GnRHa+hMG & 5 \\
\hline 29 & 39 & IVF & GnRHantagonist+hMG & 5 \\
\hline 29 & 39 & IVF & hMG & 9 \\
\hline
\end{tabular}

CC: clomiphene citrate, hMG: human menopausal gonadotrophin, GnRHa: GnRH agonist.

\section{Results}

Table 1 shows the method of inducing ovulation and the number of oocytes for each OPU in 29 women. Each woman was healthy and didn't have any complications other than infertility. The average age at the first OPU was $37.4 \pm 4.3$. To achieve fertility, conventional in vitro fertilization (IVF) and intracytoplasmic sperm injection (ICSI) were adopted for each OPU after sperm counts. Thus, among the 29 women, 20 were treated with conventional IVF only, 8 with ICSI only, and 1 with both methods. The number of oocytes in the first OPU varied from 0 to 12 , with an average of 4.83 (SD: 2.72). The average number of oocytes among several OPUs in each individual varied from one to 9.5. The average number of oocyte counts and their SDs for each patient are listed in
Figure 1. SDs of oocyte counts tended to be larger in patients with a larger number of oocytes.

Next, the ratio of SD of the oocyte counts/average oocyte counts in OPU was evaluated for each patient. As oocyte counts increased, the size of the validation itself also increased. However, looking at the ratio of SD/average count of oocytes in OPU, patients with a large number of oocyte counts have a rather small validation rate (Figure 2). Patients with more oocytes tended to have a lower ratio of SD/average oocyte count in OPU. In addition, the value of SD/average of oocyte counts in OPU was 1.41 , in patients with one oocyte as an average; however, the validation in this value was smaller in patients with a larger average oocyte count. Furthermore, we evaluated the number of patients in each validation group (Figure 3). Of the 29 patients, 7 $(24.1 \%)$ had $\mathrm{SD} \geq 2$ and $3(10.3 \%)$ had $\mathrm{SD} \geq 3$.

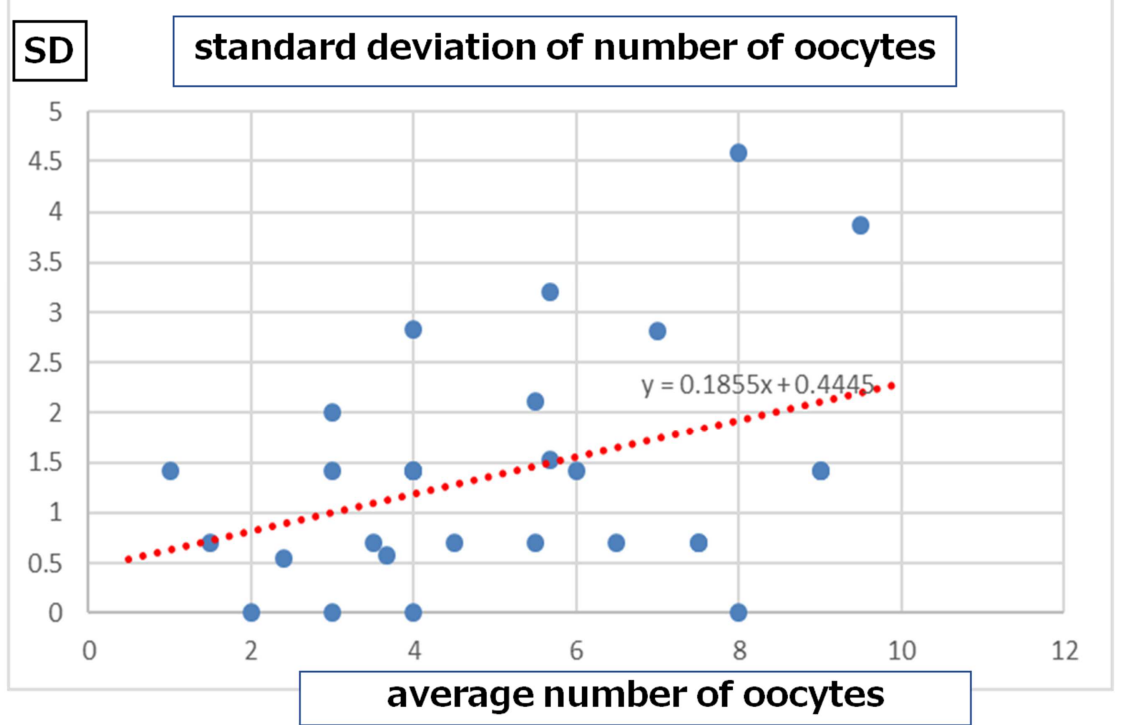

Figure 1. Standard deviation of number of oocytes increased according to larger average number of oocytes. 


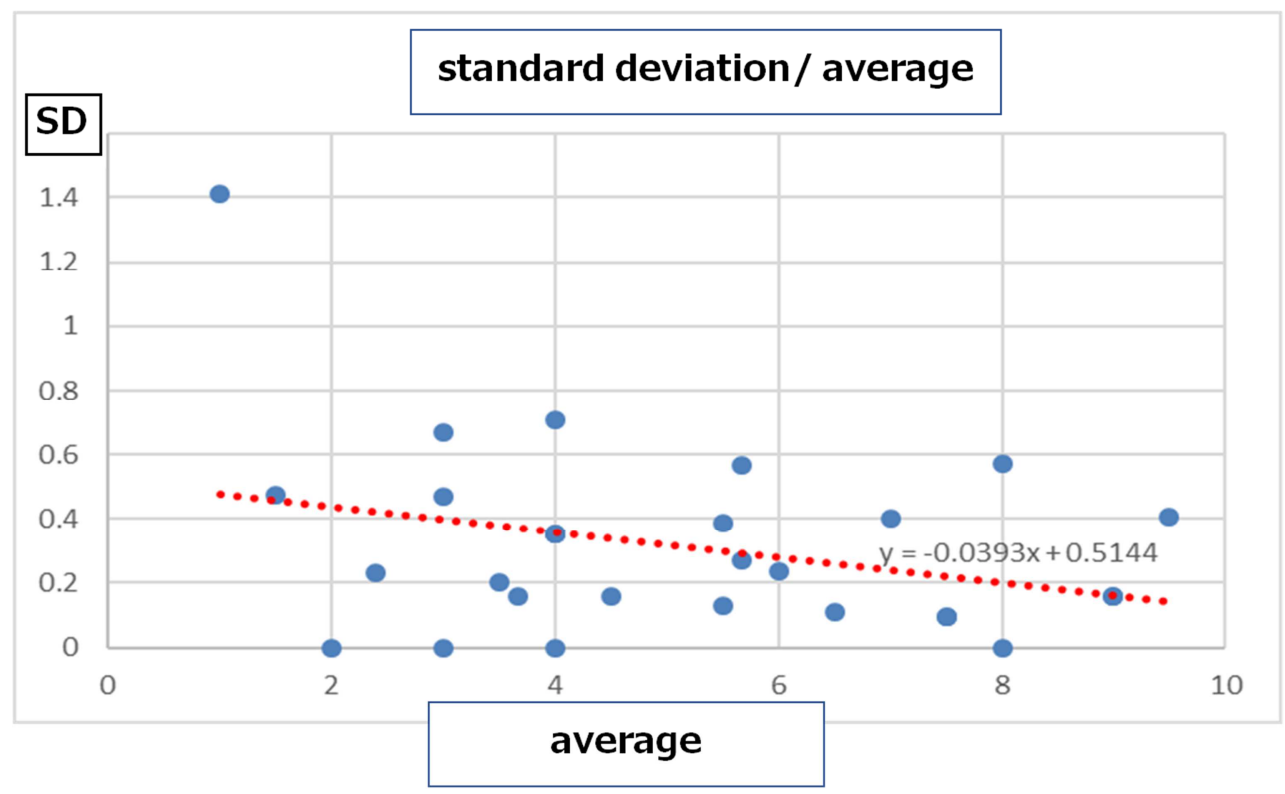

Figure 2. Ratio of standard deviation/ average decreased according as average number of oocytes increased.

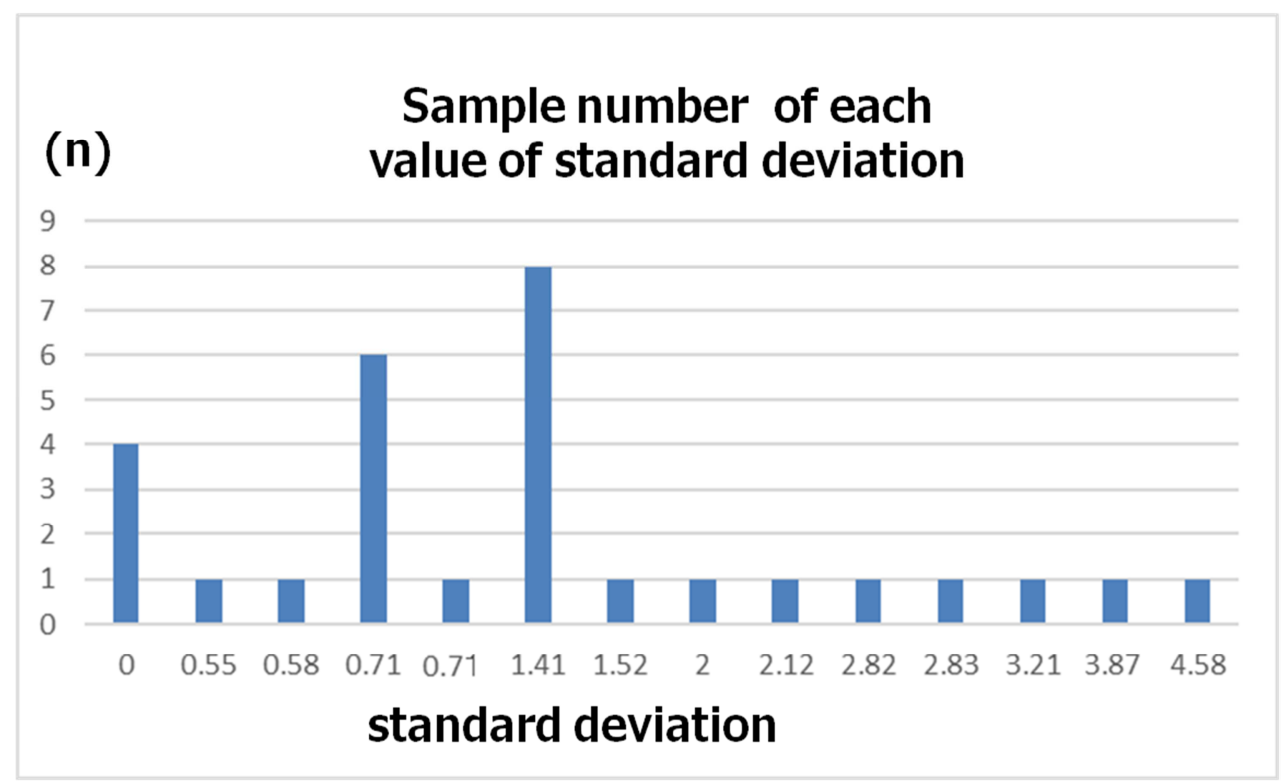

Figure 3. Sample number of each value of standard deviation is shown.

\section{Discussion}

ART has brought great benefits to women with infertility, and, in the process, AMH has played an important role as a useful test to determine the ovarian reserve. $\mathrm{AMH}$ is associated with follicle count in controlled ovarian stimulation. However, this relationship is not reliable in some cases, such as polycystic ovarian syndrome.

When a woman attempts to get pregnant by ART, there are two ways. One way is as follows. The oocytes obtained from each OPU are sequentially adopted for transfer, and if the patient fails to conceive after using up all stocked embryos, next OPU will be planned. The other way is as follows. OPU is repeated until a sufficient number of embryos are obtained. Which is a better strategy for achieve pregnancy in terms of mental and economic perspective? From our data, 29 women among 103 recieved OPU more than once. Repetition of OPU is not a rare event for pregnancy, leading to accumulation of cost $[12,13]$. Therefore, the question above is important to

Selecting a method will depend on the number of embryos required for pregnancy at each age and the estimated number of oocytes collected at one time for a certain patient. Thereafter, the frequency of OPU for the patient could be estimated. Thus, it is important to know the variation in the number of oocytes in OPU for each patient. In this study, we evaluated the variation in oocyte counts in patients with infertility who underwent multiple OPU within 1 year from the first OPU.

$\mathrm{AMH}$ can be used as a reference for predicting the counts of oocytes; however, it stands for averages and is only an indicator of ovarian reserve. In their prediction, previous 
counts may have strong values, thus its characteristic as a predictive factor for the following OPU, which this study aimed to confirm.

Despite their low oocyte count, patients suffering from infertility hope and expect larger oocyte counts after OPU due to possible individual variation. However, based on our results, oocyte counts differed between patients, and the variation was not significant.

Since oocyte counts do not change significantly, a long-term plan until pregnancy may help. In cases of successful pregnancy, if surplus embryos remain as a stock, they can be used for the next pregnancy. On the other hand, even if there is a shortage, in this case, there is no time loss as compared with the other method: re-OPU after use of all embryos. Presentation of both methods is useful information for patients.

The limit of this study was the lack of information on patients with no oocytes, including premature ovarian insufficiency. Other approaches may be needed for such patients. This research was conducted on patients who received OPU several times in 1 year; however, in the future, oocyte counts over a longer period of time should be examined. Another limitation of our research was the lack of comment on the quality of oocytes or embryos.

However, the most important factor for good embryos is age $[14,15]$. The estimated number of embryos for a successful pregnancy depends on the age; therefore, the expected value of the exact number of oocytes will help patients to know the time prior to conception, reducing the patient's physical, psychological, and financial burdens. Long-term reproductive treatments are psychologically, economically, and temporally stressful to the patient. It seems useful to consider a period of at least 1.5 years in future studies. Furthermore, our findings may be useful in estimating the oocyte counts for preserving an appropriate stock of embryos in the future and based on this result, a large-scale analysis is required. In addition, the cost of ART differs among countries. If the cost itself is open, it may be helpful for considering ART in each country.

\section{Conclusion}

The number of oocytes increased as the average number of oocytes increased. However, the ratio of standard deviation/average decreased. The number of oocytes in OPU can be a strong predictor in the following OPU in near future. In addition, this method may decrease not only economical but also mental burden from infertile women.

\section{Conflict of Interest}

All the authors have no conflict of interest.

\section{Acknowledgements}

We thank Dr. Suzuki Fumiaki for clinical support.

\section{References}

[1] G. M. Chambers, G. D. Adamson, M. J. Eijkemans, Acceptable cost for the patient and society, Fertil Steril 100 (2) (2013) 319-27.

[2] S. Iliodromiti, T. W. Kelsey, O. Wu, R. A. Anderson, S. M. Nelson, The predictive accuracy of anti-Mullerian hormone for live birth after assisted conception: a systematic review and meta-analysis of the literature, Hum Reprod Update 20 (4) (2014) 560-70.

[3] S. L. Broer, F. J. Broekmans, J. S. Laven, B. C. Fauser, Anti-Mullerian hormone: ovarian reserve testing and its potential clinical implications, Hum Reprod Update 20 (5) (2014) 688-701.

[4] N A. Dennis, L. A. Houghton, G. T. Jones, A. M. van Rij, K. Morgan, I. S. McLennan, The level of serum anti-Mullerian hormone correlates with vitamin D status in men and women but not in boys, J Clin Endocrinol Metab 97 (7) (2012) 2450-5.

[5] N. Suzuki, N. Yoshioka, S. Takae, Y. Sugishita, M. Tamura, S. Hashimoto, Y. Morimoto, K. Kawamura, Successful fertility preservation following ovarian tissue vitrification in patients with primary ovarian insufficiency, Hum Reprod 30 (3) (2015) 608-15.

[6] I. A. van Rooij, F. J. Broekmans, E. R. te Velde, B. C. Fauser, L. F. Bancsi, F. H. de Jong, A. P. Themmen, Serum anti-Mullerian hormone levels: a novel measure of ovarian reserve, Hum Reprod 17 (12) (2002) 3065-71.

[7] T. Piltonen, L. Morin-Papunen, R. Koivunen, A. Perheentupa, A. Ruokonen, J. S. Tapanainen, Serum anti-Mullerian hormone levels remain high until late reproductive age and decrease during metformin therapy in women with polycystic ovary syndrome, Hum Reprod 20 (7) (2005) 1820-6.

[8] F. Magri, L. Schena, V. Capelli, M. Gaiti, F. Zerbini, E. Brambilla, M. Rotondi, M. De Amici, A. Spinillo, R. E. Nappi, L. Chiovato, Anti-Mullerian hormone as a predictor of ovarian reserve in ART protocols: the hidden role of thyroid autoimmunity, Reprod Biol Endocrinol 13 (1) (2015) 106.

[9] B. Vollenhoven, S. Hunt, Ovarian ageing and the impact on female fertility, F1000Res 7 (2018).

[10] C. Gnoth, A. N. Schuring, K. Friol, J. Tigges, P. Mallmann, E. Godehardt, Relevance of anti-Mullerian hormone measurement in a routine IVF program, Hum Reprod 23 (6) (2008) 1359-65.

[11] Kumasawa, K., Yasui, Yuri. Nakamura, Hitomi., Kimura Tadashi. Increased Anti-mullerian Hormone (AMH) After Delivery: Case Report, Journal of Gynecology and Obstetrics 2016; 4 (3): 17-18.

[12] M. C. Inhorn, P. Patrizio, Infertility around the globe: new thinking on gender, reproductive technologies and global movements in the 21st century, Hum Reprod Update 21 (4) (2015) 411-26.

[13] L. Bahamondes, M. Y. Makuch, Infertility care and the introduction of new reproductive technologies in poor resource settings, Reprod Biol Endocrinol 12 (2014) 87.

[14] N. M. Crawford, A. Z. Steiner, Age-related infertility, Obstet Gynecol Clin North Am 42 (1) (2015) 15-25.

[15] M. V. Sauer, Reproduction at an advanced maternal age and maternal health, Fertil Steril 103 (5) (2015) 1136-43. 